\title{
Diffuse Abdominal Splenosis Mimicking Peritoneal Metastases in a 35-Year-Old Man with a Resectable Carcinoma of the Ampulla of Vater
}

\author{
Steffen Filskov Sorensen ${ }^{a} \quad$ Frank Viborg Mortensen $^{\mathrm{b}} \quad$ Ylva Hellberg $^{\mathrm{c}}$ \\ Morten Ladekarl ${ }^{a}$ \\ Departments of ancology and ${ }^{b}$ Gastrointestinal Surgery, and ${ }^{c}$ Institute of Pathology, \\ Aarhus University Hospital, Aarhus, Denmark
}

\section{Key Words}

Ampullary carcinoma $\cdot$ Splenosis $\cdot$ Peritoneal metastasis

\begin{abstract}
A 35-year-old man with a history of blunt abdominal trauma and splenic rupture was diagnosed with an ampullary adenocarcinoma. At workup, a CT scan showed multiple intraabdominal lesions similar to peritoneal carcinosis, and the patient was referred for palliative chemotherapy. On clinical suspicion, however, a biopsy was performed on an intraabdominal lesion, establishing the diagnosis of abdominal splenosis. A radical pancreaticoduodenectomy ad modum Whipple was performed, followed by adjuvant chemotherapy with gemcitabine. At the 18-month follow-up, the patient was free from recurrent disease. We conclude that splenosis should be considered as a differential diagnosis of peritoneal metastases in cancer patients with a history of abdominal trauma and/or splenectomy. Other reports on splenosis in cancer patients and diagnostic workup are discussed.
\end{abstract}

(C) 2013 S. Karger AG, Basel

\section{Introduction}

Usually, multiple tumor-like lesions observed in the abdominal cavity in a patient with an ampullary cancer would be indicative of peritoneal dissemination and, consequently, of incurable disease. As illustrated by the following case, a rare benign differential diagnosis to 
peritoneal metastases should always be considered in the absence of other signs of inoperability.

\section{Case Report}

A 35-year-old man had suffered from intermittent upper abdominal pain for approximately 1 month. An ultrasound of the abdomen revealed no pathology, and routine blood tests were normal apart from an elevated P-amylase of $374 \mathrm{U} / \mathrm{l}$ (approx. twice the upper normal limit). At elective gastroscopy 3 weeks later, the patient reported increasing fatigue and an unintended weight loss of $7 \mathrm{~kg}$. On clinical examination, he was icteric. His bilirubin was $105 \mu \mathrm{mol} / \mathrm{l}$ (normal range 5-25 $\mathrm{mol} / \mathrm{l}$ ), ALAT was $621 \mathrm{U} / \mathrm{l}$ (normal range 10-70 U/l) and alkaline phosphatase was $430 \mathrm{U} / \mathrm{l}$ (normal range 35-105 U/l). Slight anemia of 7.2 $\mathrm{mmol} / \mathrm{l}$ (normal range $8.3-10.5 \mathrm{mmol} / \mathrm{l}$ ) and thrombocytosis of $424 \times 10^{9} / \mathrm{l}$ (normal range $\left.145-350 \times 10^{9} / 1\right)$ were also found. The patient's medical report included splenectomy following a motocross accident with blunt abdominal trauma and intra-abdominal bleeding 20 years earlier, and surgery for appendicitis the same year.

The gastroduodenoscopy showed a macroscopically malignant tumor located between the first and second part of the duodenum, involving almost the entire lumen. Biopsies showed an invasive, poorly differentiated adenocarcinoma of the intestinal type. A CT scan with intravenous and peroral contrast (fig. 1) revealed a tumor in the ampulla (arrow) involving the whole circumference of the duodenal lumen, but with no apparent involvement of major blood vessels or surrounding organs. In addition, multiple nodular tumors were seen in several intra-abdominal locations, giving rise to the suspicion of peritoneal metastases (fig. 2). The nodules varied in size from a few millimeters up to $60 \mathrm{~mm}$ (A arrows). Surgical clips from the patient's prior splenectomy were also seen (B arrow). After these findings, the patient was referred to the multidisciplinary team conference for discussion of palliative chemotherapy.

At the multidisciplinary team conference, the patient's medical history and signs of previous surgery, including absence of the spleen and presence of multiple surgical clips in the abdominal wall, as well as the uncharacteristic appearance of the intra-abdominal lesions, raised the suspicion of diffuse posttraumatic splenosis. In support of this diagnosis, the CT scan showed slightly hypervascular lesions, with soft tissue attenuation and homogeneous enhancement in the portal venous phase. A core needle biopsy of 1 lesion showed characteristic morphological and immunohistological appearances of benign ectopic splenic tissue.

A radical pancreaticoduodenectomy ad modum Whipple was performed following laparoscopy. The presence of splenosis was confirmed macroscopically and with frozen biopsies during surgery. The resected tumor was a poorly differentiated adenocarcinoma, originating from the papilla of Vater, with intravascular and perineural growth. Two of 22 lymph nodes contained metastatic adenocarcinoma. The TNM stage was pT2 pN1 M0 R0.

The postoperative CA 19-9 was normal (2.4 IU/l). After discussing the option with the patient, adjuvant treatment was offered consisting of gemcitabine $1,000 \mathrm{mg} / \mathrm{m}^{2}, 3$ weeks on, 1 week off, for 6 months. He tolerated the treatment well and is without any signs of recurrence at follow-up, 18 months after surgery. 


\section{Discussion}

Splenosis, first named by Buchbinder and Lipkoff [1], is a condition observed after splenectomy, especially following traumatic rupture of the spleen. The location of the autotransplanted implants is limited only by anatomical boundaries. However, hematogenous spread has also been reported, resulting in intrahepatic and intracranial sites of implantation, and thoracic splenosis has been reported in cases having both a rupture of the spleen and the diaphragm [2-5]. Splenosis occurs in up to $65 \%$ of patients with traumatic or iatrogenic splenic rupture with an average delay of 10 years to presentation (range 5 months to 32 years), but the incidence of splenosis in the general population is unknown. The number of implants is related to the severity of the splenic injury. Patients with splenosis are mostly asymptomatic and the abnormality is often found incidentally [6].

The diagnosis of splenosis can be established noninvasively by a radionuclide scintigraphic study of the liver and spleen with Technetium (Tc)-99m sulfur colloid. A more sensitive and specific diagnosis can be made by Tc-99m-radiolabelled heat-damaged erythrocytes or indium 111-labeled platelets [7, 8]. SPECT-CT fusion imaging based on Tc$99 \mathrm{~m}$ sulfur colloid can be used to give supplemental three-dimensional anatomical information [8, 9]. Ferumoxides-enhanced MRI, in which superparamagnetic iron oxide particles administered intravenously are removed from the circulation by the reticuloendothelial system of the liver and spleen and subsequently visualized by MRI, is not a fully established modality since it is expensive and there are few published data [10]. CT and ultrasound are nonspecific imaging modalities with respect to splenosis. CT can be used to characterize the number, shape, size and location of the nodules, but a more specific imaging modality or a biopsy must be performed subsequently to establish the diagnosis. Direct biopsies from the spleen are often avoided due to the risk of bleeding, but a biopsy from a suspected splenic implant is believed to be safe, since the blood supply of implants differs from the spleen proper.

The mimicking of splenosis and peritoneal metastases has been described previously. Short et al. [9] described a 60-year-old man who was suspected of having metastatic disease from an unknown primary tumor after the finding of multiple intra-abdominal nodules on a CT scan. A Tc-99m sulfur colloid scintigraphy and SPECT-CT scan were performed, and a biopsy confirmed the diagnosis of splenosis. In another case report by Kang et al. [2], a 54year-old man, who previously was splenectomized due to an abdominal trauma, was initially diagnosed with a gastric cancer and a solitary liver metastasis. The workup consisted of a PET/CT scan on which no signs of metastasis were seen; however, an abdominal ultrasound revealed a tumor in the liver. Superparamagnetic iron oxide liver MRI was performed. The tumor had high signal intensity on T2-weighted images and low signal intensity on T1weighted images, raising the suspicion of intrahepatic splenosis. A radical gastrectomy and a liver resection were performed, and the histopathological examination revealed that the lesion suspected to be a liver metastasis was a splenosis implant.

Splenosis may be confused with abdominal lymphoma, endometriosis, simple lymphadenopathy, primary tumors (including renal, gastric or hepatic tumors) and secondary metastatic disease including carcinomatosis, peritoneal mesothelioma and liver metastases $[6,11,12]$. Clearly, the described concurrent diagnosis of splenosis and ampullary adenocarcinoma in our 35-year-old patient is exceptional, and, furthermore, the patient had numerous, widely scattered lesions, which is unusual. However, due to the catastrophic consequences of misdiagnosis, splenosis should always be ruled out in cancer patients with a history of abdominal trauma and/or splenectomy if the malignancy is otherwise resectable. 
Sorensen et al.: Diffuse Abdominal Splenosis Mimicking Peritoneal Metastases in a 35 Year-Old Man with a Resectable Carcinoma of the Ampulla of Vater

\section{Acknowledgements}

We thank Prof. Steven Hamilton for language editing.

\section{Disclosure Statement}

The authors have no conflicts of interest to declare.

\section{References}

1 Buchbinder JH, Lipkoff CJ: Splenosis: multiple peritoneal splenic implants following abdominal injury. Surgery 1939;6:927-934.

2 Kang KC, Cho GS, Chung GA, et al: Intrahepatic splenosis mimicking liver metastasis in a patient with gastric cancer. J Gastric Cancer 2011;11:64-68.

3 Rickert CH, Maasjosthusmann U, Probst-Cousin S, August C, Gullotta F: A unique case of cerebral spleen. Am J Surg Pathol 1998;22:894-896.

4 Yammine JN, Yatim A, Barbari A: Radionuclide imaging in thoracic splenosis and a review of the literature. Clin Nucl Med 2003;28:121-123.

5 Normand JP, Rioux M, Dumont M, Bouchard G, Letourneau L: Thoracic splenosis after blunt trauma: frequency and imaging findings. AJR Am J Roentgenol 1993;161:739-741.

6 Fremont RD, Rice TW: Splenosis: a review. South Med J 2007;100:589-593.

-7 Gunes I, Yilmazlar T, Sarikaya I, Akbunar T, Irgil C: Scintigraphic detection of splenosis: superiority of tomographic selective spleen scintigraphy. Clin Radiol 1994;49:115-117.

$>8$ Schillaci O, Filippi L, Danieli R, Simonetti G: Single-photon emission computed tomography/computed tomography in abdominal diseases. Semin Nucl Med 2007;37:48-61.

-9 Short NJ, Hayes TG, Bhargava P: Intra-abdominal splenosis mimicking metastatic cancer. Am J Med Sci 2011;341:246-249.

$\longrightarrow 10$ Berman AJ, Zahalsky MP, Okon SA, Wagner JR: Distinguishing splenosis from renal masses using ferumoxide-enhanced magnetic resonance imaging. Urology 2003;62:748.

11 Priola AM, Picciotto G, Priola SM: Diffuse abdominal splenosis: a condition mimicking abdominal lymphoma. Int J Hematol 2009;90:543-544.

12 Abu Hilal M, Harb A, Zeidan B, Steadman B, Primrose JN, Pearce NW: Hepatic splenosis mimicking HCC in a patient with hepatitis $\mathrm{C}$ liver cirrhosis and mildly raised alpha feto protein; the important role of explorative laparoscopy. World J Surg Oncol 2009;7:1. 
Sorensen et al.: Diffuse Abdominal Splenosis Mimicking Peritoneal Metastases in a 35Year-Old Man with a Resectable Carcinoma of the Ampulla of Vater
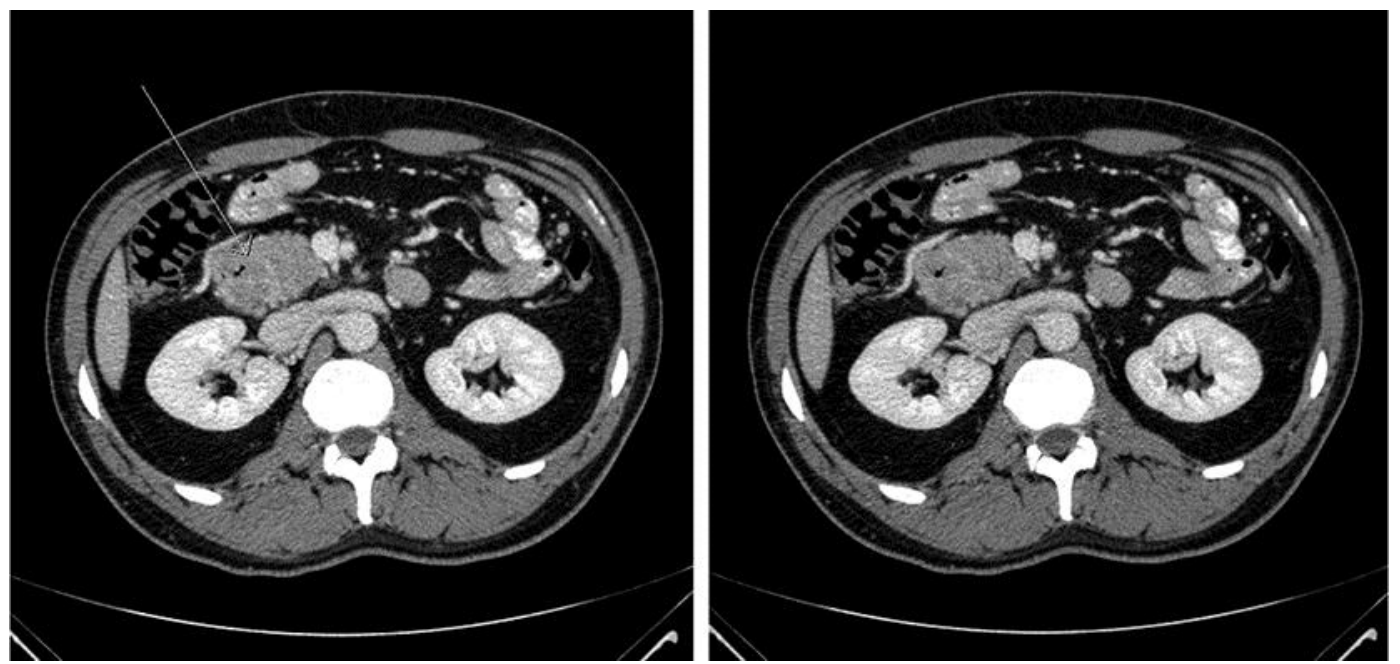

Fig. 1. CT scan of the patient with intravenous and peroral contrast. A tumor in the duodenum involving almost the entire lumen (arrow) can be seen.
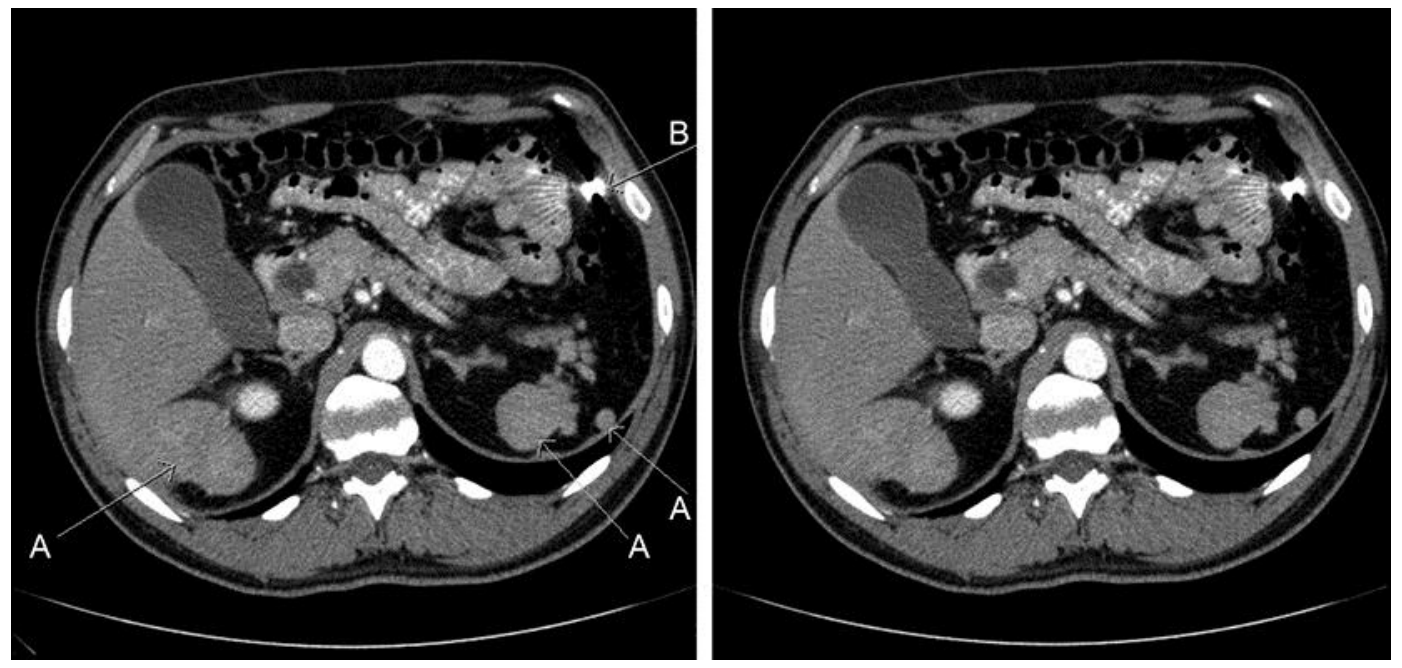

Fig. 2. Images from the same scan as in fig. 1. Multiple nodules of different sizes can be seen in different intra-abdominal locations (A arrows). Surgical clips (B arrow) are also seen as a sign of previous abdominal surgery. 